\title{
Diseño de un sistema de inventario computarizado para la rastreabilidad de partes reutilizables
}

\section{Design of a computed inventory system for the traceability of reusable parts}

\author{
ACOSTA-HERNANDEZ, Daniel $\uparrow^{*}$, DE LA RIVA-RODRIGUEZ, Jorge, WOOCAY-PRIETO, Arturo \\ y REYES-MARTÍNEZ, Rosa María
}

Instituto Tecnologico de Ciudad Juarez

ID 1er Autor: Daniel, Acosta-Hernandez / ORC ID: 0000-0001-6680-8000, CVU CONACYT ID: 963834

ID 1er Coautor: Jorge, De La Riva-Rodriguez / ORC ID: 0000-0001-8402-0065, CVU CONACYT ID: 214030

ID 2do Coautor: Arturo, Woocay-Prieto / ORC ID: 0000-0001-9235-0494, CVU CONACYT ID: 163516

ID 3er Coautor: Rosa María, Reyes-Martínez / ORC ID: 0000-0002-6433-8129, CVU CONACYT ID: 240644

DOI: $10.35429 /$ JIE.2019.9.3.18.25

Recibido 02 de Julio, 2019, Aceptado, 30 de Agosto, 2019

\begin{abstract}
Resumen
Este artículo presenta el diseño de un sistema computarizado para la rastreabilidad y reutilización de piezas, donde impacta en la reducción de costos de manufactura, contribuyendo a la disminución del consumo de recursos naturales. Los componentes a reutilizar son recuperados de un equipo completo usado, partiendo de un listado de todas sus partes. El sistema facilita la rastreabilidad de los componentes que se requieren recuperar, reduciendo el tiempo de localización y suministrando de piezas a las áreas de producción. Además, evita la saturación en el área de almacén de piezas que no son requeridas. Este sistema contara con datos en tiempo real y conciso sobre las entradas y salidas de materia prima, agilizando su recepción de los equipos, realizándose la captura del inventario de entrada. Como resultado propiciará mejorar la planificación de la producción, incrementando los índices de recuperación de los componentes requeridos, aprovechando al máximo el área de almacenamiento, disminuyendo tiempos de desembarque de los equipos. Además, permitirá un mejor costeo de entradas y salidas de la empresa. Por otra parte, el costo de desarrollo del software fue mínimo, y podría impactar de forma considerable, en las utilidades de la industria, ecología y la economía donde se implemente.
\end{abstract}

Inventario, Reciclaje, Planeacion

\begin{abstract}
This article presents the design of a computerized system for the traceability and reuse of pieces, where it impacts on the reduction of manufacturing costs, contributing to the decrease in the consumption of natural resources. The components to be reused are recovered from a complete used equipment, starting from a list of all its parts. The system facilitates the traceability of the components that need to be recovered, reducing the time of location and supplying parts to the production areas. In addition, it avoids the saturation in the warehouse area of parts that are not required. This system will have data in real time and concise on the inputs and outputs of raw material, speeding up the reception of equipment, making the capture of input inventory. As a result, it will lead to better production planning, increasing the recovery rates of the required components, making the most of the storage area, decreasing equipment unloading times. In addition, it will allow a better costing of inputs and outputs of the company. On the other hand, the software development cost was minimal, and could have a considerable impact on the profits of the industry, ecology and the economy where it is implemented.
\end{abstract}

Inventory, Recycle, Planning

Citación: ACOSTA-HERNANDEZ, Daniel, DE LA RIVA-RODRIGUEZ, Jorge, WOOCAY-PRIETO, Arturo y REYESMARTÍNEZ, Rosa María. Diseño de un sistema de inventario computarizado para la rastreabilidad de partes reutilizables. Revista de Ingeniería Industrial. 2019 3-9: 18-25

\footnotetext{
* Correspondencia del Autor (Correo electrónico: $9 @$ yahoo.com)

$\dagger$ Investigador contribuyendo como primer autor.
} 


\section{Introducción}

A medida que las industrias aumentan la demanda de productos, estas generan en sus mayoría daños al medio ambiente algunos de ellos irreversibles, por lo que se desarrolló una disciplina llamada Reciclaje, en donde se practican tres sencillos pasos: reutilizar, reciclar y recuperar (Berenguer, 2006), al llevar a cabo esto se ayuda al cuidado del medio ambiente, evitando la sobreexplotación de recursos naturales y sobre todo en el aspecto administrativo de compras en las empresas. La reducción en las compras de piezas, es lo que más impacta en la reducción de residuos, debido a que solo se compran las piezas que se necesiten y en la cantidad correcta evitando la generación de productos no requeridos. Esto ayuda considerablemente disminuir la extracción de materiales y su transformación (Abdul-Rahman, 2014). Algunas empresas optan por la reutilización de piezas debido a que algunas partes resultan ser más económicas o forman parte de sus políticas ecológicas. Esta práctica ecológica es una responsabilidad de las empresas (Wen Kam, 2016). La reutilización de piezas como política de reciclaje o de reducción de costos debe ser administrado a través de un software o sistema de inventarios para agilizar las entradas y salidas de existencias, además, se mantiene a salvo la información en caso de desastre natural o incendio. Siendo que estos registros se tienen de manera manual estos pudiesen ser dañados e irrecuperables al momento de su perdida. (Opeyemi, 2013).

La gestión del inventario cubre la planificación y control de inventarios, en donde las prioridades determinan que materiales se requieren y cuando son necesarios para cubrir la demanda del cliente, siendo la parte más esencial de las empresas para el cumplimiento de la producción. (Hong-Mo, 2018). Con un sistema confiable de inventarios se optimiza la reutilización de las partes, el cual identifique la cantidad y el tipo de parte que requiera el cliente o producción y con ello aumentar el índice de recuperación y como consecuencia las ventas. Si se conoce las partes que componen cada equipo es más fácil planificar la producción debido a que se conoce con veracidad, si existe o no la pieza recuperada. Con lo cual se realizara el suministro de piezas en las diferentes áreas de manera controlada y facilitando la medición y desempeño de la empresa. (Kumar, 2013).
Uno de los sistemas de inventario más conocido, es la Planeación de Requerimiento de Materiales (MRP), en donde este considera el control y la planeación de la producción. Con este sistema se logrará reaccionar de manera adecuada a los cambios generados por la demanda del producto, es decir ajustar al mismo tiempo el pronóstico y actividades como por ejemplo, los puntos de abastecimiento, los tamaño de lotes, los tiempos de entrega y los cambios en los inventarios (Reyes, 2011). Sin embargo, es un sistema general de inventarios y no está muy accesible para reutilización de los componentes de equipos específicos ya que cada uno estos se presentan con características diferentes. Además, las empresas requieren una considerable cantidad de información estructurada y eficiente para desempeñar las sus funciones de los distintos departamentos Fleitas (2013).

En la empresa estudiada, los equipos se reciben en plataformas, el registro y control de materiales se realizaba manualmente, lo que perjudicaba a los departamentos de producción y recepción de materiales (equipos ensamblados) debido a la falta de información de los componentes recuperados de cada uno de los equipos registrados en el inventario, afectando directamente a producción por no conocer cuántos componentes se disponían, lo que ocasionaba una respuesta lenta a las demandas del cliente. El proceso que se tenía era demasiado deficiente para conocer con rapidez y exactitud los equipos recibidos registrados en el inventario, ya que se realizaba a través de lápiz y papel, por consecuencia su captura era lento y con alto índice de error, al momento de registrar los equipos que contenían las plataformas. Las plataformas que se registraron en el año 2017 fueron un total de 52 dando un total de 2,362 bombas, 557 motores y 1,293 protectores sumando un total de 4,212 teniendo como promedio por plataforma de 81 equipos por descarga a inventariar tal y como se muestra en la siguiente tabla 1.

\begin{tabular}{|l|r|}
\hline Descripción & Cantidad recibida 2017 \\
\hline Bombas & 2.362 \\
\hline Motor & 557 \\
\hline Protector & 1.293 \\
\hline Total & 4.212 \\
\hline \multicolumn{2}{|c|}{} \\
\hline Plataformas & 52 \\
\hline
\end{tabular}

Tabla 1 Equipos Recibidos en Enero-Dic. 2017 Fuente: Fabricaciones SAGE

ACOSTA-HERNANDEZ, Daniel, DE LA RIVA-RODRIGUEZ, Jorge, WOOCAY-PRIETO, Arturo y REYES-MARTÍNEZ, Rosa María Diseño de un sistema de inventario computarizado para la rastreabilidad de partes reutilizables. Revista de Ingeniería Industrial. 2019 3-9: 18 
Para mejorar el control de inventario se desarrolló un software, en Visual Basic el cual facilita el manejo de bases de datos. Se diseñó un software sencillo de manipular, ya que permite la interacción de los datos con distintas aplicaciones de información como por ejemplos en los formatos de Word, Excel, PowerPoint y Access (Kremer, 2010). La estructura de la base de datos para este sistema descrito en este artículo consiste: lugar de procedencia de los equipos, placas de la plataforma, tipo de equipo a inventariar conforme al número de parte de equipo y la lista de componentes del equipo, para así obtener una pronta respuesta al cliente o a las líneas de producción.

El Visual Basic nos genera un archivo ejecutable que puede ser corrido en cualquier computadora libre de errores de programación (Luna, 2011). Con una estructura de programación de Visual Basic para Aplicaciones (VBA). Para el manejo de bases de datos se utilizó el Microsoft Office Access. Además, se utilizó el comando de tablas para la interacción de los datos entre sí, para la importación de datos de Excel al VBA, para el desarrollo e impresión de los diferentes reportes o visualización en la pantalla o la exportación de datos a otro programa (Support Office, 2017), entre otras herramientas que ofrece el software (VBA) de programación como lo son: consultas, Macros, Módulos y palabras reservadas para la programación.

Para la captura y manejo de los datos y el control del inventario, se realizó a través de pantallas amigables, con la finalidad de mantener actualizada las cantidades y detalles de los componentes recuperados. Lográndose con esto mantener en el almacén solo los componentes que requiere el cliente y respaldar las operaciones de producción (Porras, 2008).

También se realizó un análisis del hardware disponible para su aplicación e implementación, dicha comparación fue entre un computador de escritorio y una computadora portátil. Una vez definido tanto el software como hardware se especificó como se realizaría la interacción de los datos.

Se optó por utilizar la conexión Red para la operación del sistema lo que da flexibilidad en su operación y utilización (Assets, 2017).
Con este sistema de inventario se logró la reducción del tiempo de la captura de información de la recepción de materiales y lo cual aumento la capacidad de plataformas descargadas de los equipos a inventariar y un mejor aprovechamiento de cada metro cuadrado de almacenamiento y otros gastos directos e indirectos implicados en la manufactura (Salas, 2014). Por otra parte se aplicaron las técnicas de Estudio del Trabajo lográndose reducir los costos e incrementando la productividad y se diseñó una herramienta para el pronóstico de tiempo para la descarga de los equipos. El estudio del trabajo fue esencial para el establecimiento de procesos y para la coordinación de la producción. (Akansel, 2017).

\section{Objetivo}

Estructurar e implementar un sistema computacional para el control, localización y asignación de espacio a piezas reutilizables, asegurando los requerimientos de producción de acuerdo a la demanda de órdenes de compra, con la finalidad de reducir el tiempo de entrega de las piezas al departamento de piso.

\section{Metodología}

La preparación de dicha investigación fue realizada de la siguiente manera:

Se analizó la recepción de materia prima de cómo se realizaba antes del nuevo sistema de inventario, se presenció la descarga y registro de una plataforma con equipo a inventariar, el registro de la información era en formatos de papel y llenado con lápiz, asignándosele a cada equipo completo un número de control interno para su pronta localización, el cual este se componía de tres partes, donde en su primera posición es el número de entrada de equipo partiendo de 1 hasta $n$-numero, en segunda posición es la letra del mes en que este se registró siendo la primera letra, para los meses de con mismas iniciales se utilizó uno diferente para evitar confundirlo con otro mes, como por ejemplo en el mes de Mayo se utilizó la letra "Y", para Julio la letra "L", en Agosto "G" y por último en el mes de Octubre la letra " $\mathrm{C}$ " siendo este mes posible de confundir con un cero por ello la decisión de cambiar su estructura en esta segunda posición. En la última posición es el tipo de equipo y es asignado con la primera letra, donde solo se tienen tres tipos de equipo donde son "B" Bombas, "P" de Protector y " $M$ " de Motor.

ACOSTA-HERNANDEZ, Daniel, DE LA RIVA-RODRIGUEZ, Jorge, WOOCAY-PRIETO, Arturo y REYES-MARTÍNEZ, Rosa María Diseño de un sistema de inventario computarizado para la rastreabilidad de partes reutilizables. Revista de Ingeniería Industrial. 2019 3-9: 18 
Se analizó el flujo de producción y su número de control para la localización, se determinaron los datos esenciales para el manejo y captura de información del sistema siendo la: procedencia de la carga o plataforma, fecha de llegada, placas de plataforma, numero de plataformas, número de ensamble del equipo, número de serie y por ultimo las condiciones del mismo, para la facilitación y búsqueda de componente en el sistema. Con la información anterior se procedió a la estructuración de las cinco bases datos

\section{Diseño del Menú Principal}

Se realizó un análisis de las necesidades de información de las áreas involucradas en el control de inventarios, las cuales pudieron ser agrupadas en cinco apartados referentes a la materia prima que son: recepción, inventario, componentes posibles a fabricar, salida, y catálogo de materia prima. Se diseñó una pantalla que contiene cinco botones que dan acceso a los cinco rublos mencionados anteriormente (Fig. 1). Los cuales se pueden activar de acuerdo a las necesidades de la empresa y cada botón están protegido con un control de accesos donde solo el personal autorizado puede realizar modificaciones $\mathrm{o}$ registros en sus secciones de almacén para proteger la información.

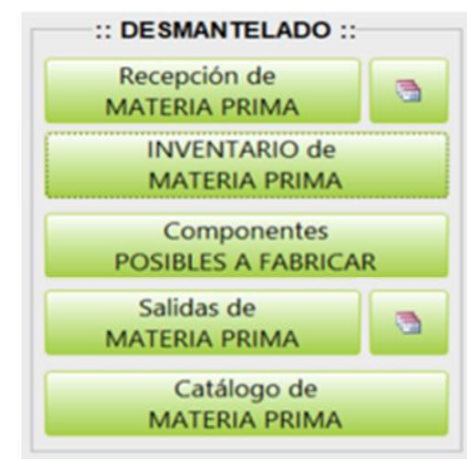

Figura 1 Secciones del Sistema

A continuación, se describirán los cinco rubros que componen la pantalla principal:

\section{Recepción de Materia Prima}

Esta sección se utiliza para registrar el equipo completo que contiene una plataforma y los datos capturados son: procedencia de carga (Plataforma), fecha de llegada, placas de plataforma, numero de plataforma, número de ensamble del equipo, número de serie del equipo, condiciones del equipo y número interno.
Esta pantalla tiene la capacidad de adjuntar archivos o imágenes del equipo requeridos por el cliente en la parte inferior con nombre "Path archivo de fotos" y "Path archivo Documentos" ver Figura (Fig. 2).

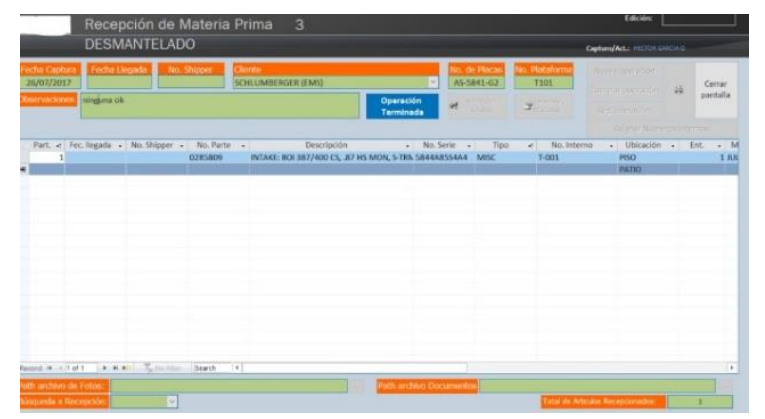

Figura 2 Recepción de Materia Prima Fuente: Elaboración Propia

\section{Inventario de materia prima}

En esta sección del sistema es requerido para la visualización de lo que se tiene en existencia en almacén de equipos completos para la recuperación de partes, en donde en ella se puede realizar una búsqueda por número de ensamble del equipo, tipo de equipo, número interno, fecha de llegada o en su defecto algún componente en específico (Fig. 3).

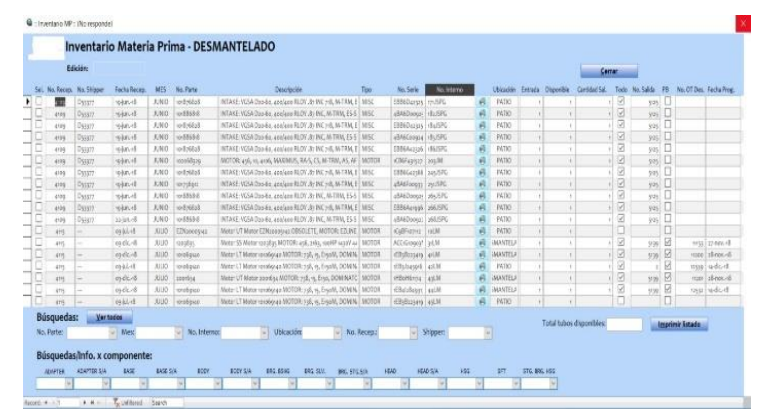

Figura 3 Inventario Disponible en Patio Fuente: Elaboración Propia

\section{Componentes Posibles a Fabricar}

En esta sección del sistema es el concentrado total de las partes contenidas en los equipos completos, que pudieran ser recuperadas y utilizadas en la planeación de producción de acuerdo con las necesidades del cliente. La pantalla facilita la búsqueda de la pieza que se quiere localizar, arrojando el plano para verificar si el componente cumple con las especificaciones del cliente (Fig.4). 


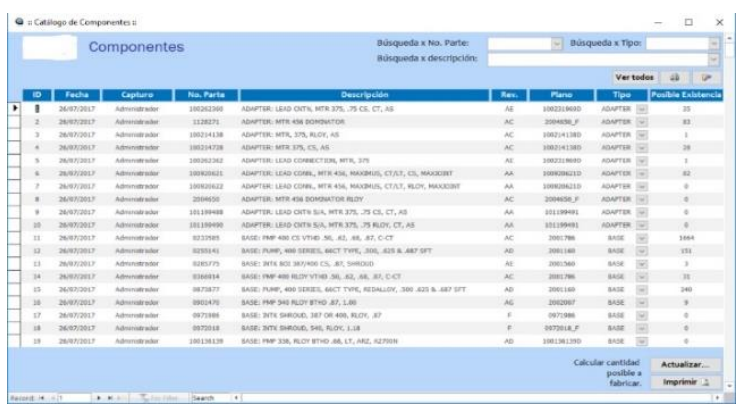

Figura 4 Componentes posibles a fabricar Fuente: Elaboración Propia

\section{Salida de Materia Prima}

La dinámica de esta sección es similar a la recepción de materia prima solo que en esta se maneja la salida del material antes registrado, dicha actividad es realizada por el supervisor de producción de desmantelado, cuando se genera una orden de compra por un o algunos componentes, los equipos son desmantelados con la finalidad de recuperar los componentes deseados. Al finalizar el proceso se requiere remover su estatus de disponibilidad en el almacén, siendo esta una actividad importante para evitar problemas al momento de realizar la planeación de la producción (Fig.5).

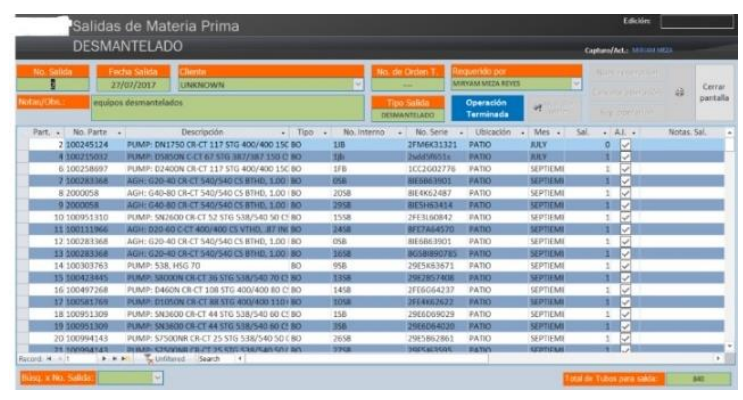

Figura 5 Salida de Materia Prima Fuente: Elaboración Propia

\section{Catálogo de Materia Prima}

Esta sección del sistema es una de las más fundamentales e importantes, ya que contiene el listado de todos los posibles equipos completos y sus componentes que pudiera ser requerido por el cliente. Antes de dar de alta un equipo en la recepción de materia prima contar con toda la información mencionada anteriormente. Este catálogo debe ser controlado y administrado de manera cuidadosa, ya que de ser modificado sin autorización ocasionaría problemas a la hora de realizar la captura de recepción del equipo o a la planeación de producción. En esta sección se encuentra el número de ensamble con cada una de las partes internas que lo componen (Fig.6).

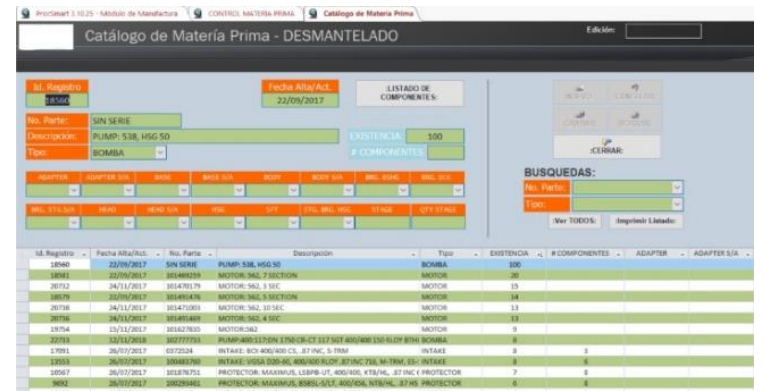

Figura 6 Catalogo de Materia Prima

Fuente: Elaboración Propia

\section{Reportes}

Como en todas las empresas la disminución de tiempos en actividades industriales son importantes pero estas deben estar sustentadas por información válida para su aprobación de los distintos departamentos de la empresa. Para la obtención de reportes de producción o de los equipos en cada una de las pantallas de este sistema computarizado pueden exportarse en distintos formatos para la manipulación externa del mismo, siendo posible su envió de datos a clientes y operar dichos datos a otros formatos: PDF, Excel, archivo de texto, Word RTF, Access, XML y HTML.

\section{Herramienta para el pronóstico de tiempo para la realización del inventario}

El cálculo de la determinación del pronóstico del tiempo de descarga de una plataforma e inventariarla fue realizado con herramientas del Estudio del trabajo lo cual permite determinar el tiempo estándar requerido para cada una de las actividades del proceso para el inventariar y registrar, y así pronosticar el tiempo de descarga según la cantidad de equipo que contenga la plataforma. Se realizó una tabla para la determinación del tiempo de descarga de las plataformas que arriban a la empresa no siempre contiene la misma cantidad de equipo teniendo variaciones constantes (Tabla 2).

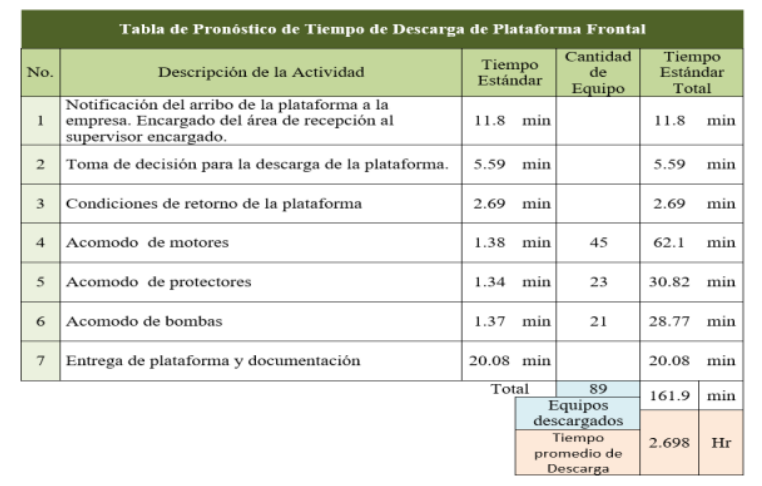

Tabla 2 Pronóstico de Tiempo de Descarga de Plataforma Frontal

\section{Resultados}

Beneficios generados del control implementado (Sistema de control nuevo).

ACOSTA-HERNANDEZ, Daniel, DE LA RIVA-RODRIGUEZ, Jorge, WOOCAY-PRIETO, Arturo y REYES-MARTÍNEZ, Rosa María Diseño de un sistema de inventario computarizado para la rastreabilidad de partes reutilizables. Revista de Ingeniería Industrial. 2019 3-9: 18 
El nuevo proceso, en el aspecto de los gastos administrativos se eliminó a un ingeniero supervisor (costo semanal de \$5,000) y se le dio la responsabilidad a un operario calificado (líder de área de recepción de materiales, con costo de $\$ 2,500$ semanales) siendo sus funciones la captura o alimentación de los datos de los equipos al sistema, y como se redujo en un $50 \%$ el tiempo en realizar estas actividades se le asignaron otras actividades en el departamento de recepción de materiales de reciente creación.

En la reducción de personal se optó por capacitar al operador (montacargistas) para realizar el acomodo del material en patio, para el flujo de las líneas de desmantelado y así quitar por completo el personal (líder de área de Desmantelado, costo semanal de \$2,000) dedicado a estas tareas anteriormente, haciendo una reducción monetaria del $100 \%$ del costo implicado a la operación, siendo este un ahorro de $\$ 2,000$ pesos semanales, teniendo en si un ahorro monetario de $\$ 4,500$ semanales $(\$ 5,000+$ $\$ 2,000=\$ 7,000-\$ 2,500=\$ 4,500$ ).

En el aspecto de la localización de las partes, antes se tomaba mucho tiempo, debido a que se desmantelaba bajo la experiencia del supervisor, suponiendo que la parte que pedían se encontraba en cierto equipo por prueba y error, ocasionando tener ocupando espacio en almacén de piezas sin órdenes de compra o requeridas por producción, además de no tener la seguridad de contar con la pieza al momento que la empresa le comunica al cliente la existencia de la misma, para que el cliente genere la orden de compra a la empresa. Con el nuevo sistema de inventario se puede verificar si realmente se tiene esa pieza, con la seguridad de surtir la orden del cliente. El tiempo de inventariado de descarga se redujo en un $45 \%$ siendo este un tiempo promedio de 3 horas menos al anterior proceso de descarga siendo este de 7 horas (disposición de plataforma) debido a las actividades que se mejoraron por el control implementado. Esto permite conocer más rápido que se tiene disponible para recuperar, y aunado a esto se aumentó la capacidad de plataformas recibidas de una o dos (sistema viejo) a 4 o 5 (sistema nuevo, comparación de plataformas y sistemas con cantidades de equipo iguales).

Además se eliminaron los papeles o formato de inventario, el cual era llenado manualmente, y este se cambió por la captura a través de una tableta electrónica la cual es un $85 \%$ más confiable, ya que con este sistema se está eliminado la diversidad de letras y numero la cual era confuso al momento de capturar y sobre todo la posible pérdida de documentos del inventario, creando la confusión entre el departamento y el cliente, cuando se aseguraba haber enviado un cierto equipo y no concordaba con el inventariado por uno o varios dígitos.
Este con el desarrollo de esta investigación, la cual se generó una herramienta de gran apoyo para la toma de decisiones de producción y pronóstico de ventas para el tiempo que se quiera obtener, tal y como se obtuvo en la tabla de pronóstico de tiempo estándar para la descarga de plataformas, de la cual es una herramienta que se desarrolló con la finalidad de obtener agilizado el área pudiendo aplicar y desarrollar el recurso más importante para las empresas el cual es el tiempo, y con dicha tabla se pronostica la tarea a desempeñar y así la disminución de costos de espera de material para la empresa como plataformas con cargas.

En cuestiones monetarias, para la gerencia se redujeron los costos de operación ya que como anteriormente se mencionó, se requería de un ingeniero para dicha operación pero con esto se logró eliminar el costo y se agilizó la rapidez en conocer algún componente en específico, y con estos datos se realiza la comparación de lo que vendió(ventas al clientes) con respecto al costo de la plataforma y en su defecto la generación de reportes de las plataformas recibidas por mes o semana llevando en si un control de ellos a través de éste sistema. Dichas ventajas y resultados anteriormente descritos, con este sistema desarrollado, se obtiene el pronóstico del tiempo requerido para tener la carga lista para su proceso (material disponible para desmantelar y recuperar) así como también los equipos que llegaron como sus componentes disponibles para su re manufactura, y así obtener respuestas concretas hacia las demanda de los clientes, siendo el sistema una herramienta de planificación de la producción como de respuesta hacia el cliente trabajando con lo demandado y evitar espacios ocupados con piezas sin órdenes de compra previstas.

Con la utilización del sistema se tuvo un registro de 7,571 equipos en el periodo EneroDiciembre del 2018 con un total de 5,227 bombas, 1,087 motores y por último en protectores de 1,257 y teniendo como registro de 105 plataformas para los equipos (2018), en comparación del 2017 (52 plataformas) como se muestra en la tabla 3 teniendo como aumento en su capacidad a la hora de recepción de los equipos. Anteriormente se llevaba un registro de estos datos en Excel provocando inconvenientes cuando el archivo se corrompía ocasionando problemas en la contabilidad de equipo comprado para producción.

\begin{tabular}{|l|r|r|}
\hline Descripción & $\begin{array}{c}\text { Cantidad recibida } \\
2017\end{array}$ & $\begin{array}{c}\text { Cantidad } \\
\text { recibida 2018 }\end{array}$ \\
\hline Bombas & 2.362 & 5.227 \\
\hline Motor & 557 & 1.087 \\
\hline Protector & 1.293 & 1.257 \\
\hline Total & 4.212 & 7.471 \\
\hline Plataformas & 32 & 105 \\
\hline
\end{tabular}

Tabla 3 Equipos Recibidos en Enero- Dic. 2017 y EneroDic. 2018

ACOSTA-HERNANDEZ, Daniel, DE LA RIVA-RODRIGUEZ, Jorge, WOOCAY-PRIETO, Arturo y REYES-MARTÍNEZ, Rosa María Diseño de un sistema de inventario computarizado para la rastreabilidad de partes reutilizables. Revista de Ingeniería Industrial. 2019 3-9: 18 
Uno de las aportaciones adicionales del sistema es la información que la proporciona en tiempo real y confiable para el desarrollo de las actividades administrativas y productivas. Por ejemplo ahora cuenta con información del comportamiento de diferentes departamentos como son; del departamento de ventas (índice de recuperación, respuesta inmediata al cliente), compras (Cantidades de plataformas en el mes), costos de maquinados o recuperación (reducción de costos) y el de producción (capacidad de desmantelado). Lo que permite realizar una planificación más certera acorde a la demanda del cliente. La Tabla 4 presenta un listado de algunos beneficios logrados con el sistema.

\begin{tabular}{|c|c|}
\hline \multicolumn{2}{|c|}{ Beneficios Obtenidos de la Investigación } \\
\hline Administrativo & Producción \\
\hline $\begin{array}{l}\text { Control de entradas y salidas de } \\
\text { material }\end{array}$ & $\begin{array}{l}\text { Capacidad de desmantelado } \\
\text { diario/ semanal }\end{array}$ \\
\hline Cantidades de plataformas en el mes & Confiabilidad en la información \\
\hline $\begin{array}{l}\text { Reducción de costos en el } \\
\text { departamento }\end{array}$ & Sistema fácil de utilizar \\
\hline Respuesta inmediata al cliente & Planificación de la producción \\
\hline Mayor estructura organizacional & Confiabilidad en almacén \\
\hline \multirow[t]{2}{*}{$\begin{array}{l}\text { Reducción de tiempo al momento de } \\
\text { inventariar }\end{array}$} & Reportes de materia prima \\
\hline & $\begin{array}{l}\text { Listado de componentes posibles } \\
\text { a recuperar }\end{array}$ \\
\hline $\begin{array}{l}\text { anancias con re: } \\
\text { ión, y sobre tod } \\
\text { la reutilizac }\end{array}$ & $\begin{array}{l}\text { cto a los efectos del s } \\
1 \text { cuidado del medio a } \\
\text { de piezas. }\end{array}$ \\
\hline
\end{tabular}

Tabla 4 Beneficios Obtenidos de la Investigación Fuente: Fabricaciones SAGE

Estos beneficios antes mencionados fueron a partir de la implementación del sistema, ya que aumentaron las ventas debido al incremento en el índice de recuperación, por lo que impactó en las satisfacciones del cliente entregándose a tiempo las demandas requeridas, se tiene mayor control de lo inventariado. Por otra parte, las líneas de producción se tiene trabajando solo con los productos que son demandados o que tiene una orden de compra y en el almacén la diminución de piezas que no tienen orden de compra, teniendo en si una mejoras en las áreas con una mayor limpieza y orden, aunado a eso y lo más importante en las investigación es la aportación que se realiza al medio ambiente por la práctica del reciclaje de piezas.

\section{Agradecimiento}

A la empresa Fabricaciones SAGE, Instituto Tecnológico de Ciudad Juarez y al departamento de Posgrado del mismo Tecnológico mencionado se le agradece por el apoyo, disponibilidad y confianza en la realización de este proyecto de investigación de impacto ecológico y económico para la empresa.

\section{Conclusión}

Al momento de realizar la investigación se detallaron $\mathrm{y}$ discutieron las necesidades $\mathrm{y}$ beneficios que se esperaban del sistema, lográndose el impacto esperado en paralelo se desarrolló otro módulo de control para una área critica de producción "Flechas" ya que debido al aumento de producción demanda por el cliente las necesidades de control fue creciendo para el mayor y mejor manejo de información en los almacenes de materia prima tal como se muestra en Figura 7.

Con el desarrollo de las tablas (Tabla 2 Pronóstico de Tiempo de Descarga de Plataforma Frontal) se obtuvieron beneficios de los cuales ayudaron al departamento de planeación y logística para la realización de maniobra y disponibilidad de las plataformas.

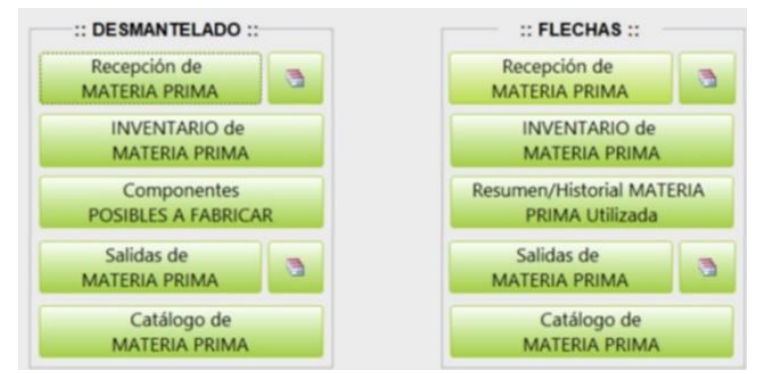

Figura 7 Modulo Nuevo (Flechas) de Inventario a partir de las Bases de Desmantelado

Fuente: Elaboración Propia

Y en el aspecto ecológico, este tipo de investigación es importante llevar acabo su réplica para las distintas ramas industriales, con la finalidad de la reducción de contaminantes al medio ambiente ayudando en si a mantener el ecosistema en equilibrio por la reutilización de piezas re-manufacturadas, siendo siempre este un objetivo de toda empresa "cuidar del medio ambiente".

\section{Sugerencias de Investigación}

Para otras investigaciones se pudiera adicionar información adicional, como agregar el valor de cada equipo), lo que ayudaría a tener una contabilidad más detallada, por ejemplo el tipo de maquinaria y/o herramientas que se requieren, consumibles, tiempos, costos directos e indirectos y para el área de manufactura, planos y hojas de inspección, u otra información que se requiera para la extracción piezas. 
Con dicha investigación antes presentada se pueden aplicar en otras áreas donde se pueda y se requiera llevar un control de inventarios añadiéndole el rubro de calidad para cada componente obtenido o posible a recuperar agregando los documentos de control de calidad tales como hojas de inspección, control y actualización de planos. En producción se puede establecer los máximos y mínimos del tipo de herramientas y equipo requerido para el re trabajo de las piezas y contar con la materia prima que genere la mayor ganancia a la empresa o evitar paros de producción por la carencia de algún componente.

\section{Referencias}

Salas, 2014. GASTOS INDIRECTOS DE PRODUCCION Disponible en: https://www.uv.mx/personal/alsalas/files/2014/ 09/GASTOS-INDIRECTOS-DE-

PRODUCCION.pdf

[Consultado el 10 de Diciembre de 2018].

Fahzy Abdul-Rahman, 2014. REDUCE, REUSE, RECYCLE: ALTERNATIVES FOR WASTE MANAGEMENT. Guide-314 New México State University.. Cooperative Extension Service, College of Agricultural, Consumer and Environmental Sciences

Hui Wen Kam, 2016. A review of commercial waste recycling policy in Malaysia. Department of Building Surveying, Faculty of Built Environment, University of Malaysia. International Journal of Environment and Sustainable Development 15(4):

Abisoye Opeyemi, At all, 2013. Design of a Computerized Inventory Management System for Supermarkets. Federal University of Technology, Department of Computer Science, Minna, Niger State, Nigeria. International Journal of Science and Research (IJSR) 2(9):2319-7064

Prasanta Kumar Dey, At all, 2013. Green supply chain performance measurement using the analytic hierarchy process: a comparative analysis of manufacturing organisations. Aston Business School, Aston University, Birmingham Production Planning and Control 25(9): I-I

Mehmet Akansel, At all, 2017. Determination of Standard Times for Process Improvement: A
Case Study. , Uludag University, Department of Industrial Engineering, Bursa 16059. Global Journal of Business, Economics and Management: Current Issues Volume 00, Issue 0, (2017) 000-000

Michael Kremer. (2010). Database Automation Using VBA UC Berkeley Extension. Sitio web: www.ucb-access.org: access@ ucb-access.org

Berenguer M, et al. (2006). EL RECICLAJE, LA INDUSTRIA DEL FUTURO. ISSN: 10272887

Denny Hong Mo Yeh, 2018. Inventory Management MGT2405, University of Toronto, Denny Hong-Mo Yeh

Dr. Primitivo Reyes Aguilar (2011). Planeación de requerimientos de materiales (MRP). Enero 2011 Ciudad de México. Sitio web: www.icicm.com/files/MRP.doc

Paula Fleitas. (2013). Access 2013 Guía Práctica para el Usuario. Buenos Aires: Fox Andina; Dalaga: Manuales USERS. ISBN: 978-9871949-17-5

RootStock Cloud ERP. (2018). Cloud ERP Software for Manufacturing, Distribution \& Supply Chain. Abril 2017, de RootStock Cloud ERP Sitio web: https://www.rootstock.com/

Eric Porras, Rommert Dekker. (2008). An inventory control system for spare parts at a refinery: An empirical comparison of different re-order point methods. En European Journal of Operational Research (101-132). ScienceDirect: Elsevier.

Fernando O. Luna. (2011). Visual Basic Guía Definitiva del Programador. Buenos Aires: Fox Andina; Dalaga: Manuales Users ISBN: 978987-1773-57-2.

Support Office. (2010). Database basics. Junio 2017, de Microsoft Sitio web: https://support.office.com/enus/article/database-basics-a849ac16-07c7-4a319948-3c8c94a7c204\#top.

Rene Ríos, José R. Fermin. (2009). Traffic Analysis of a University local Network. Revista Electrónica de Estudios Telemáticos, 8, 13. ISSN: 1856-4194 